\title{
Image of the Month: Clinical Features in a Newborn with Covered Cloacal Exstrophy
}

\author{
Alejandra Vilanova-Sánchez ${ }^{1}$ Christina B. Ching ${ }^{2}$ Alessandra C. Gasior ${ }^{3}$ Karen Diefenbach ${ }^{3}$ \\ Richard J. Wood ${ }^{4}$ Marc Levitt ${ }^{4}$
}

${ }^{1}$ Department of Pediatric Surgery, Center for Colorectal and Pelvic Reconstruction, Nationwide Children's Hospital, Columbus, Ohio, United States

2 Department of Pediatric Urology, Nationwide Children's Hospital, Columbus, Ohio, United States

${ }^{3}$ Department of Pediatric Surgery, Nationwide Children's Hospital, Columbus, Ohio, United States

${ }^{4}$ Center for Colorectal and Pelvic Reconstruction, Nationwide

Children's Hospital, Columbus, Ohio, United States

Address for correspondence Alejandra Vilanova-Sánchez, Department of Pediatric Surgery, Hospital Universitario La Paz, 611 Livingston Avenue, Columbus, $\mathrm{OH}$ 43206, United States (e-mail: vilanova.alejandra@gmail.com).

Eur J Pediatr Surg Rep 2017;5:e57-e59.

\author{
Abstract \\ Keywords \\ - covered cloacal \\ exstrophy \\ - variant \\ - anorectal \\ malformation \\ - bladder duplication
}

Cloacal exstrophy is the most severe type of anorectal malformations that belongs to the bladder-exstrophy-epispadias complex of genitourinary malformations. Interestingly, its variant, the covered cloacal exstrophy, is often missed. The clinical findings of this variant may include an imperforate anus, low lying umbilicus, thick pubic bone, and pubic diastasis but with an intact abdominal wall. We present an interesting case of covered cloacal exstrophy with a side-by-side duplicated bladder and discuss important considerations for the time of colostomy creation in the newborn period.

\section{New Insights and the Importance for the Pediatric Surgeon}

To recognize a covered cloacal exstrophy as early as possible. One must have a high clinical suspicion in a patient with an anorectal malformation and a low-set umbilicus, a pubic diastasis, short colon, and vesicocolonic fistula. Correct diagnosis directs further evaluation of other urologic malformations and enables proper surgical management, including saving as much colon as possible by removing the colon from the bladder at the time of colostomy formation. This improves the potential for future continence by maximizing the child's ability to form solid stool. Ultimately, a combined urologic and colorectal reconstruction can be performed.

\section{Introduction}

Covered cloacal exstrophy is a rare congenital malformation with many different anatomic variants. Given the intact abdominal wall, this diagnosis can be missed despite having severe intra-abdominal anatomic abnormalities (e.g., abnormal and shortened colon, colonicurinary tract communication) that resemble those observed in true cloacal exstrophy 1 .
There are distinct clinical and radiologic features (i.e., imperforate anus, low lying umbilicus, pubic diastasis, foreshortened colon) observed in these infants; however, a high clinical suspicion is required to make an accurate diagnosis and properly treat the patient in the newborn period. We present a patient treated at our institution who also had covered cloacal exstrophy and a side-by-side duplicated bladder with single urethra and phallus. received

July 29, 2017

accepted

August 1, 2017
DOI https://doi.org/

10.1055/s-0037-1606389.

ISSN 2194-7619. (c) 2017 Georg Thieme Verlag KG Stuttgart · New York
License terms

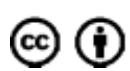




\section{Case Presentation}

A newborn of 36 weeks of gestation with karyotype XY presented to our institution with an imperforate anus and passing stool from a hypospadic urethra. He had a single phallus with a coronal hypospadias, penile shaft lipoma, and complete separation of his hemiscrotum. In addition, it was noted that he had a low lying umbilicus and a pubic diastasis ( - Fig. 1).

He was taken to the operating room at 24 hours of life for abdominal exploration and planned colostomy. At this time, a fistula from the rectum to the dome of the bladder was observed. The colon was noted to be short with only the appearance of a right colon and two appendices. The vesicocolonic fistula was taken down and the bladder primarily repaired. The mobilized colon was matured into an end colostomy in the left mid quadrant. VACTERL screening revealed a tethered cord, a sacral ratio of 0.6 , multiple vertebral anomalies, side-by-side duplication of his bladder (with a single ureter entering each bladder found upon retrograde pyelogram), and proximal $\mathrm{Y}$ duplication of his urethra (-Fig. 2). He underwent urodynamic testing of each bladder that showed low pressure, compliant bladders of low-volume capacity on each side. He had normal appearing kidneys on renal ultrasound without hydronephrosis, which were normally located in the right and left renal fossa. He had a normal creatinine level of 0.23 . He was able to generate a urinary stream but was still in diapers. There have been no urinary tract infections (UTIs) since 1 year of age. The stoma effluence is thickening and he is feeding well and gaining weight.

\section{Discussion}

The clinical features we present are compatible with covered cloacal exstrophy: low lying umbilicus, pubic diastasis, short colon, vesicocolonic fistula, and other urologic abnormalities. ${ }^{1}$ Covered cloacal exstrophy is a rare disease with many different anatomic variants. The initial diagnosis of exstrophy variants

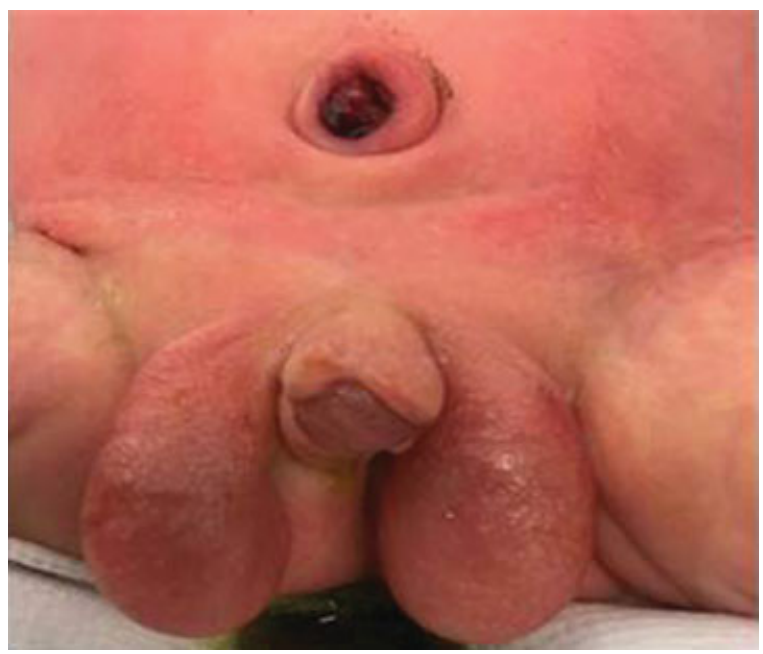

Fig. 1 Male newborn with clinical features of covered cloacal exstrophy: pubic bone diastasis, low lying umbilicus, and meconium in urine (rectourinary tract fistula).

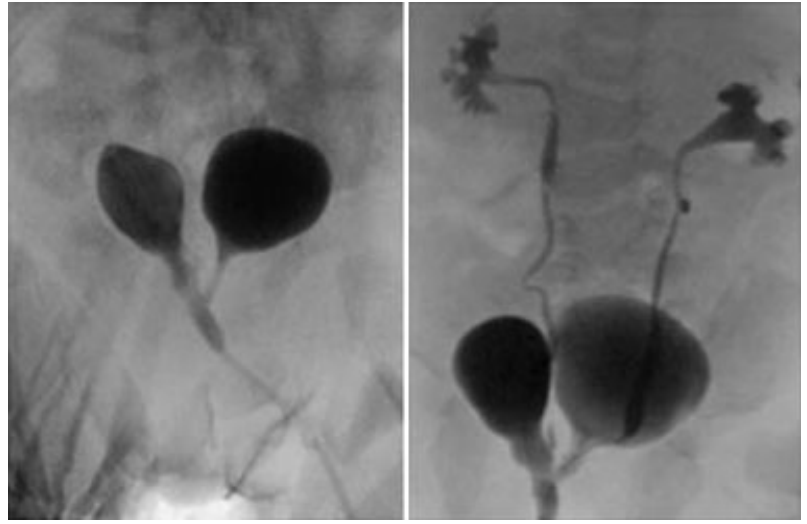

Fig. 2 Retrograde pyelogram showing a side-by-side duplication of the bladder with a single ureter entering each bladder in a patient with covered cloacal exstrophy.

can be difficult because their presentation can be confusing. The resulting delay in initial treatment can have significant consequences in surgical planning and future continence outcomes. $^{2} \mathrm{~A}$ high clinical suspicion is required to make the diagnosis and may include a prenatal ultrasound suggestive of classic cloacal exstrophy (such as low-set umbilicus, omphalocele, diastasis of the pubic rami, split vulva, unilateral renal agenesis). The presence of bladder filling and absence of the characteristic elephant trunk appearance of the prolapsing terminal ileum may suggest the diagnosis of a covered cloacal exstrophy prenatally. ${ }^{3,4}$ In addition, these patients often commonly have severe spinal abnormalities, such as a tethered cord or spinal dysraphisms. ${ }^{5}$

With regard to colorectal management, it is extremely important to diagnose the malformation in the newborn period, as this will affect the management of the bowel during stoma creation. The goal should be to maintain as much colon as possible, and include it in the fecal stream, including separation of bowel as low as possible from the urinary tract, to improve the potential to make solid stool and a future pull through. ${ }^{6}$

With regard to urologic management, it is important to screen for other urologic abnormalities and consider the potential for neurogenic pathology, ultimately affecting bladder function. This patient had an unusual associated urologic malformation of a side-by-side duplicated bladder. The significance of these bladders was unclear, as he was clinically well and not near toilet training age. Side-by-side bladder duplications may in fact be continent, as each bladder can have its own intact sphincter. ${ }^{2}$ He would need repair of his genitalia (hypospadias, scrotoplasty, and removal of the penile shaft lipoma). He is planned to have a pull through of the colostomy with access for antegrade flushes at toilet training age. Clearly close collaboration between colorectal surgery and pediatric urology is the key to providing this child optimal care. ${ }^{7}$

\section{Conflict of Interest}

None. 


\section{References}

1 Bischoff A, Levitt MA, Breech L, Peña A. Covered cloacal exstrophy-a poorly recognized condition: hints for a correct diagnosis. J Pediatr Surg 2013;48(12):2389-2392

2 Lowentritt BH, Van Zijl PS, Frimberger D, Baird A, Lakshmanan Y, Gearhart JP. Variants of the exstrophy complex: a single institution experience. J Urol 2005;173(05):1732-1737

3 Ono K, Kikuchi A, Takagi K, Takahashi D, Yoshizawa K, Nishizawa S. Prenatal sonographic features of complete covered cloacal exstrophy. Ultrasound Obstet Gynecol 2009;34(04):481-482
4 Mallmann MR, Reutter H, Müller A, et al. Prenatal diagnosis of covered cloacal exstrophy. Fetal Diagn Ther 2014;36(04):333-336

5 Johnston JH, Koff SA. Covered cloacal exstrophy: another variation on the theme. J Urol 1977;118(04):666-668

6 Levitt MA, Mak GZ, Falcone RA Jr, Peña A. Cloacal exstrophy-pullthrough or permanent stoma? A review of 53 patients. J Pediatr Surg 2008;43(01):164-168, discussion 168-170

7 Lakshmanan Y, Bellin PB, Gilroy AM, Fung LC. Antenatally diagnosed cloacal exstrophy variant with intravesical phallus in a twin pregnancy. Urology 2001;57(06):1178 